Check for updates

Cite this: Chem. Sci., 2019, 10, 2349

¿ All publication charges for this article have been paid for by the Royal Society of Chemistry

Received 14th August 2018

Accepted 30th December 2018

DOI: $10.1039 / c 8 s c 03624 a$

rsc.li/chemical-science

\section{Lysine carbonylation is a previously unrecognized contributor to peroxidase activation of cytochrome $c$ by chloramine- $\mathrm{T} \uparrow$}

\author{
Victor Yin, (D) Safee H. Mian and Lars Konermann (D) *
}

The peroxidase activity of cytochrome $c$ (cyt $c$ ) plays a key role during apoptosis. Peroxidase catalysis requires a vacant Fe coordination site, i.e., cyt $c$ must undergo an activation process involving structural changes that rupture the native Met80-Fe contact. A common strategy for dissociating this bond is the conversion of Met80 to sulfoxide (MetO). It is widely believed that this MetO formation in itself is sufficient for cyt $c$ activation. This notion originates from studies on chloramine-T-treated cyt $c$ (CTcyt $c$ ) which represents a standard model for the peroxidase activated state. CT-cyt $c$ is considered to be a "clean" species that has undergone selective MetO formation, without any other modifications. Using optical, chromatographic, and mass spectrometry techniques, the current work demonstrates that CTinduced activation of cyt $c$ is more complicated than previously thought. MetO formation alone results in only marginal peroxidase activity, because dissociation of the Met80-Fe bond triggers alternative ligation scenarios where Lys residues interfere with access to the heme. We found that CT causes not only MetO formation, but also carbonylation of several Lys residues. Carbonylation is associated with -1 Da mass shifts that have gone undetected in the CT-cyt c literature. Proteoforms possessing both MetO and Lys carbonylation exhibit almost fourfold higher peroxidase activity than those with MetO alone. Carbonylation abrogates the capability of Lys to coordinate the heme, thereby freeing up the distal site as required for an active peroxidase. Previous studies on CT-cyt c may have inadvertently examined carbonylated proteoforms, potentially misattributing effects of carbonylation to solely MetO formation.

\section{Introduction}

Cytochrome $c$ (cyt $c$ ) is a $12 \mathrm{kDa}$ mitochondrial heme protein whose primary role is to serve as an electron shuttle in the respiratory chain. ${ }^{1}$ Cyt $c$ also possesses a second biologically relevant function as a peroxidase. ${ }^{2-8}$ Peroxidases catalyze the oxidation ( $\mathrm{H}$ abstraction) of substrates in the presence of $\mathrm{H}_{2} \mathrm{O}_{2}$. Catalytic turnover involves heme cycling between the Fe(III) and $\mathrm{Fe}(\mathrm{Iv})=\mathrm{O}$ states. ${ }^{9}$ The peroxidase activity of cyt $c$ is critical for triggering apoptosis (programed cell death) via oxidation of the mitochondrial membrane lipid cardiolipin., ${ }^{7 \mathbf{1 0 - 1 2}}$ Apoptosis is an essential process, e.g., for suppressing tumor growth. ${ }^{\mathbf{1 3}}$

Peroxidases require a pentacoordinated heme with a vacant distal coordination site where $\mathrm{H}_{2} \mathrm{O}_{2}$ can bind, and where the $\mathrm{Fe}(\mathrm{Iv})=\mathrm{O}$ oxygen can be accommodated. ${ }^{\mathbf{1 4}}$ The heme in native cyt $c$ is hexacoordinated, as the distal site is occupied by the Met80 sulfur (Fig. 1). ${ }^{15}$ Dissociation of this Met80-Fe contact is a mandatory prerequisite for converting cyt $c$ into a peroxidase. ${ }^{\mathbf{1 4 , 1 6 , 1 7}}$ There are different ways to achieve displacement of the

Department of Chemistry and Department of Biochemistry, The University of Western Ontario, London, Ontario, N6A 5B7, Canada.E-mail: konerman@uwo.ca

$\dagger$ Electronic supplementary information (ESI) available. See DOI: $10.1039 / \mathrm{c} 8 \mathrm{sc} 03624 \mathrm{a}$ distal Met80, such as unfolding, ${ }^{14,16}$ chemical, ${ }^{5,17-20}$ or mutational modifications, ${ }^{4,7,18,21-26}$ as well as cardiolipin binding. ${ }^{3,27-29}$

Exposure to basic $\mathrm{pH}$ induces the formation of "alkaline" conformations where Lys72, 73 or 79 serve as distal ligands instead of Met80..$^{20,30-34}$ Chemical or mutational modifications can shift this alkaline transition into the neutral range..$^{5,19,20,35-40}$

We recently demonstrated ${ }^{\mathbf{4 1}}$ that $\mathrm{H}_{2} \mathrm{O}_{2}$ converts native cyt $c$ into an active peroxidase via a reaction sequence that starts with Tyr67 oxidation, followed by dissociation of the Met80-Fe contact. Subsequently, Lys72 or 73 occupy the distal coordination site. Carbonylation then abrogates the capability of Lys72/ 73 to serve as Fe ligands, thereby generating the free distal site that is required for an active peroxidase. ${ }^{\mathbf{4 1}}$ Lys carbonylation corresponds to the oxidation of the $\varepsilon$-amino group to an aldehyde (LysCHO, aminoadipic semialdehyde, $\Delta M=-1 \mathrm{Da}){ }^{\mathbf{4 2 , 4 3}}$

The most straightforward method to rupture the Met80-Fe bond is via Met oxidation to methionine sulfoxide (MetO). It is widely believed that this oxidative modification is sufficient for generating the open distal site that is required for peroxidase activation of cyt $c$ in vitro and in vivo..$^{17,18,37,38,44-47}$ In other words, this Met80-centric view envisions a simple causal relationship between MetO formation and peroxidase activity. ${ }^{17,18,37,38,45-47}$

A key pillar of the Met80-centric view comes from work on chloramine-T (CT) oxidized cyt $c$ (CT-cyt $c$ ). CT is a mild oxidizing 
A

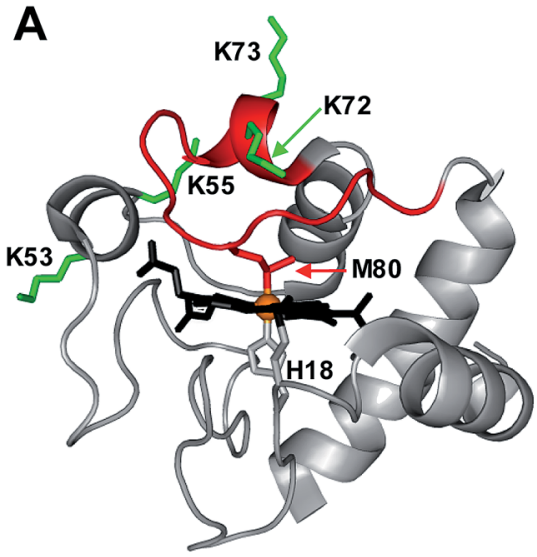

B

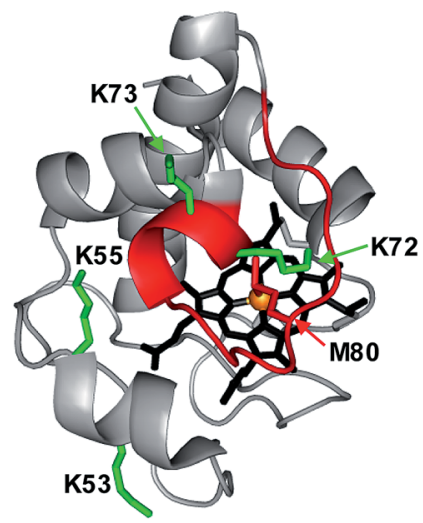

Fig. 1 Crystal structure of equine cyt $c(1 \mathrm{HRC})$ with key residues highlighted. (A) Side view. (B) Top view. In the native state, the heme co-factor is ligated by Met 80 and His18. Lys72/73 and Lys53/55 represent possible alternative distal ligands that may interact with Fe instead of Met 80 under certain conditions. The flexible $71-85 \Omega$ loop is shown in red.

agent $t^{48}$ that transforms into other reactive species in aqueous solution. ${ }^{49}$ Among these, $\mathrm{OCl}^{-}$appears to be mainly responsible for cyt $c$ oxidation, evident from the fact that cyt $c$ exposure to CT and to $\mathrm{OCl}^{-}$causes similar effects. ${ }^{17}$ CT-cyt $c$ is a potent peroxidase. ${ }^{17,37}$ Several studies have shown that CT-cyt $c$ carries a sulfoxide at both Met80 and Met65, while claiming that no other chemical modifications are present. ${ }^{17,48,50}$ These claims go back to amino acid analyses conducted in the 1970s, which concluded that CT specifically oxidizes Met, while leaving all other residues unmodified (except for -SH groups, which are absent in cyt $c$ ). ${ }^{51}$ Those early studies ${ }^{17,48,50,51}$ cemented the widely held belief that Met80 oxidation, without any other covalent changes, generates an active peroxidase. ${ }^{17,18,37,45-47}$ As a result, CT-cyt $c$ has become a widely used model for peroxidase-activated cyt $c{ }^{17,37,38,47}$

Considering the far-reaching implications of work in this area (including the role of cyt $c$ in apoptosis and tumorigenesis) ${ }_{,}^{13}$ it is imperative to fully understand the properties of CTcyt $c$. Is it true that Met80 oxidation is sufficient for peroxidase activation? Is it true that MetO formation is the only CT-induced oxidative modification? Several lines of evidence hint at the involvement of additional factors. (1) Disruption of the Met80Fe bond tends to produce "alkaline" ligation scenarios, where even at near-neutral pH the distal site becomes occupied by side chains such as Lys72/73. ${ }^{19,35,36,38}$ These alternative ligation scenarios are promoted by the flexible nature of the 71-85 $\Omega$ loop (Fig. 1)..$^{52,53}$ Hence, it is not obvious why Met80 oxidation in itself would produce a vacant distal site that is required for peroxidase activity. (2) The purported strict selectivity of CT for Met is somewhat suspicious, considering that other oxidants can induce various modifications at different types of residues. ${ }^{54-58}$ (3) Typical CT-cyt $c$ literature protocols utilize strong cation exchange (SCX) chromatography, which separates proteins according to their positive surface charge. Surprisingly, CT-cyt $c$ undergoes SCX fractionation into several distinct bands. ${ }^{37,38,47,48}$ MetO formation does not directly affect charge. The fact that CT-cyt $c$ produces several SCX fractions thus suggests that the protein might contain additional, hitherto unidentified modifications. The possible existence of such modifications and their implications for peroxidase activation have been ignored thus far in the literature.

In this work we critically examined the structure and function of CT-cyt $c$. Various mass spectrometry (MS) techniques were applied to characterize CT-induced covalent modifications in unprecedented detail. We were able to pinpoint previously unidentified features of the peroxidase activation process. Met80 oxidation alone was found to result in low peroxidase activity, because the distal site remained impeded by alkalinelike Lys ligation. Only after opening of the distal site by CTinduced LysCHO formation did the protein show higher levels of activity. Previous studies on CT-cyt $c$ that assumed a "clean" MetO protein have-in all likelihood-inadvertently characterized samples that contained both MetO and LysCHO sites. Our findings argue against the prevailing notion that Met80 oxidation alone is sufficient for peroxidase activation. Instead, Lys carbonylation is a key peroxidase co-activator.

\section{Methods}

\section{Materials}

Horse heart ferri-cyt $c, \mathrm{H}_{2} \mathrm{O}_{2}$, guaiacol (o-methoxyphenol), ammonium acetate, and chloramine-T ( $N$-chloro-4-toluolsulfonamide), were from Sigma Aldrich (St. Louis, MO). Solvents were from Fisher Scientific (Nepean, ON). Potassium phosphate was supplied by Caledon Laboratories (Georgetown, ON), and MS-grade modified trypsin was from Promega (Madison, WI). All experiments were conducted at $22 \pm 2{ }^{\circ} \mathrm{C}$.

\section{Preparation of CT-cyt $c$}

Treatment of cyt $c$ with CT was performed as described, ${ }^{37}$ with minor alterations. Briefly, a $5 \mathrm{mM}$ aqueous solution of CT was added to $1 \mathrm{mM}$ cyt $c$ (both in $50 \mathrm{mM}$ Tris buffer, $\mathrm{pH}$ 8.4) in a $1: 1$ volume ratio and reacted at room temperature for 3 hours. The reaction was then halted by dialysis against $10 \mathrm{mM}$ potassium phosphate (pH 7.4) using 10 kDa MWCO filters (EMD Millipore, Etobicoke, ON). The CT-cyt $c$ produced in this way was either 
used directly, or subjected to SCX chromatography using a $5 \mathrm{~mL}$ HiTrap sulfopropyl (SP) cartridge (GE Healthcare) on an ÄKTApurifier FPLC. The HiTrap SP column was conditioned as per manufacturer instructions, and proteins were loaded at 0.25 $\mathrm{mL} \min ^{-1}$. The column was then washed with $\mathrm{H}_{2} \mathrm{O}$ until the absorbance $(280 \mathrm{~nm}$ ) reached the pre-loading baseline. SCX elution was performed using a linear gradient of $500 \mathrm{mM}$ ammonium acetate $(\mathrm{pH} 9)$ over 5 column volumes while collecting the eluent in $1 \mathrm{~mL}$ fractions.

\section{Optical spectroscopy}

UV-Vis spectra were acquired on a Cary-100 spectrophotometer (Varian, Mississauga, ON). Soret measurement were performed on $10 \mu \mathrm{M}$ protein solutions in $50 \mathrm{mM}$ potassium phosphate buffer (pH 7.4). Absorbance measurements at $695 \mathrm{~nm}$ were performed at $40 \mu \mathrm{M}$ protein. Peroxidase activity was measured as described ${ }^{59}$ by tracking the oxidation of $9 \mathrm{mM}$ guaiacol in the presence of $100 \mu \mathrm{M}$ $\mathrm{H}_{2} \mathrm{O}_{2}$. Additional experiments were conducted using $500 \mu \mathrm{M}$ $\mathrm{H}_{2} \mathrm{O}_{2}$. This $\mathrm{H}_{2} \mathrm{O}_{2}$ concentration range is in line with previous work, ${ }^{7,32}$ and it is comparable to physiological $\mathrm{H}_{2} \mathrm{O}_{2}$ levels at the onset of apoptosis. ${ }^{60}$ In these assays the formation of tetraguaiacol was probed by UV-Vis at $470 \mathrm{~nm} \cdot{ }^{59}$ All activity assays contained 1 $\mu \mathrm{M}$ protein as determined by absorption measurements at the Soret maximum. Kinetic measurements at each $\mathrm{H}_{2} \mathrm{O}_{2}$ concentration were repeated in triplicate. Circular dichroism (CD) spectra were recorded on a Jasco J-810 spectropolarimeter (JASCO, Easton, $\mathrm{MD}$ ), on $10 \mu \mathrm{M}$ protein solutions in phosphate buffer ( $\mathrm{pH} 7.4$ ).

\section{Mass spectrometry}

MS experiments were performed on a Synapt G2 ESI quadrupoletime-of-flight instrument (Waters, Milford, MA) operated in resolution mode. The capillary, sample cone, and extraction cone voltages were set at $+2.8 \mathrm{kV}, 25 \mathrm{~V}$, and $4 \mathrm{~V}$, respectively. MS/MS experiments were performed using collision-induced dissociation (CID) with argon as collision gas. Protein samples for bottom-up MS experiments were prepared by overnight digestion using trypsin (50:1 protein/trypsin) at $37^{\circ} \mathrm{C}$, and subsequently analyzed by liquid chromatography-mass spectrometry (LC/MS) using a nanoACQUITY UPLC (Waters) with a C18 column. Samples for intact protein MS were prepared as $10 \mu \mathrm{M}$ solutions in a $50: 50$ solution of $\mathrm{H}_{2} \mathrm{O}: \mathrm{ACN}$ with $0.1 \%$ formic acid, and directly infused at $5 \mu \mathrm{L} \min ^{-1}$ using a syringe pump. For ion mobility-assisted top-down MS/MS experiments (top-down CIDIM-MS), 16+ protein ions were quadrupole-selected prior to CID in the trap collision cell. The collision energy used was $23 \mathrm{~V}$ for all samples. Ion mobility spectrometry (IMS) separation of CID products was performed in $\mathrm{N}_{2}$ buffer gas at a wave velocity of $350 \mathrm{~m} \mathrm{~s}^{-1}$ and wave height of $13 \mathrm{~V}$. Simulated isotope distributions were generated using ProteinProspector (UCSF). All simulated spectra correspond to the ferric (Fe(III)) state of cyt $c$.

\section{Results and discussion}

\section{Chloramine-T-induced Met80 oxidation}

The CT-cyt $c$ used in this study was prepared using established procedures. ${ }^{37,38,47}$ An initial characterization was conducted by subjecting unfractionated samples to a standard tryptic digestion/LC-MS/MS workflow. As expected, these assays revealed MetO formation (+16 Da) at Met80 and Met65, in line with earlier reports (Fig. $\mathrm{S} 1 \dagger$ )..$^{\mathbf{1 7 , 3 7 , 4 6 , 4 7}}$ No other +16 Da modifications were detected. These observations appear to support the widely held belief that CT selectively oxidizes Met. ${ }^{17,48,50,51}$ However, from the data discussed below it will be seen that standard mapping experiments (such as those of Fig. S1 $\dagger$ ) provide an incomplete view of the CT-induced oxidation events.

\section{SCX fractionation and optical characterization}

SCX elution profiles of CT-cyt $c$ exhibited three major peaks (Fig. 2A), consistent with previous observations..$^{37,38,47,48}$ Close examination of the highest intensity region revealed the presence of two partially overlapping signals (around $23 \mathrm{~mL}$, equivalent to "peak B" in ref. 37). In total, therefore, SCX revealed the existence of four chromatographically distinguishable CT-cyt $c$ forms. We will refer to these fractions as I, II, III, and IV in the order of decreasing retention. Fraction I had the largest retention volume, i.e., it interacted most strongly with the stationary phase, implying the most highly cationic character. The SCX behavior of fraction I was indistinguishable from that of unmodified cyt $c$. Conversely, fraction IV showed
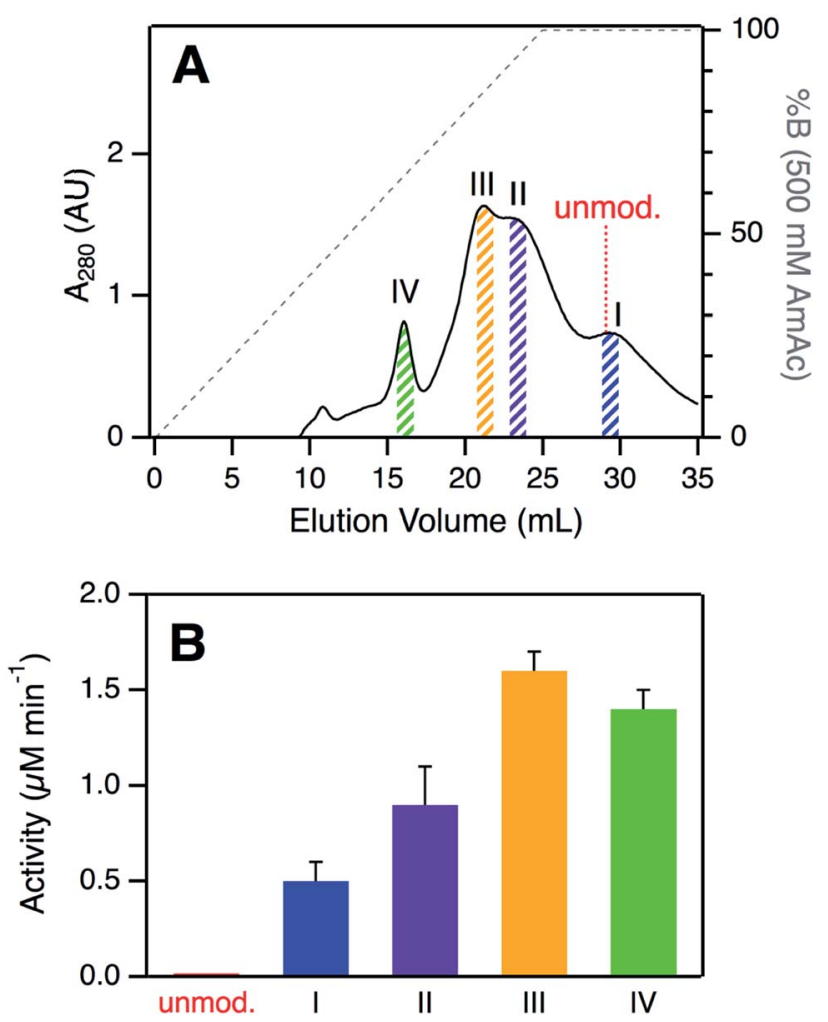

Fig. 2 (A) Analysis of CT-cyt c by SCX chromatography. Four major species (I-IV) were discernible. Colored bars indicate collected fractions used for subsequent experiments. The elution volume of unmodified cyt $c$ is indicated for comparison. (B) Peroxidase activity of CT-cyt $c$ fractions measured in $100 \mu \mathrm{M} \mathrm{H}_{2} \mathrm{O}_{2}$. See Fig. S3A and ESI Table $\mathrm{S} 1 \uparrow$ for more information. Error bars represent standard deviations of three independent experiments. 
the weakest interaction with the stationary phase, i.e., proteins in this fraction carried the least amount of positive charge. From the $A_{280}$ values in the SCX chromatograms, the relative abundance of fractions IV, III, II, and I can be estimated to be roughly $10 \%, 35 \%, 35 \%$, and $20 \%$, respectively. For subsequent experiments we isolated thin slices out of the chromatograms for targeted experiments on each fraction (Fig. 2A).

Optical spectra of the four CT-cyt $c$ fractions were very similar to one another, but markedly different from those of unmodified cyt $c$ (Fig. S2 $\dagger$ ). CT-cyt $c$ showed a $\sim 30 \%$ CD intensity loss at $222 \mathrm{~nm}$, revealing some decrease of $\alpha$-helicity upon oxidation. ${ }^{61}$ There was no concomitant increase in the random coil region ( $200 \mathrm{~nm}$ ), suggesting that CT-induced modifications did not cause unfolding, but transitions to alternative folded structures. ${ }^{61}$ A blue shift of the UV-vis Soret band from $409 \mathrm{~nm}$ to $\sim 405 \mathrm{~nm}$ indicated alterations in the heme environment. In addition, all CT-cyt $c$ fractions exhibited loss of the $695 \mathrm{~nm}$ absorption band, signaling disruption of the Met80-Fe bond. ${ }^{62}$

In summary, the spectroscopic data of Fig. $\mathrm{S} 2 \uparrow$ indicate the formation of non-native, structured conformers in the CTtreated samples. The CD spectra were similar to those of Lysligated alkaline cyt $c{ }^{\mathbf{1 9 , 3 5 , 3 6}}$ The blue-shifted Soret band is reminiscent of alkaline cyt $c$ as well. ${ }^{34,38}$ Resonance Raman (RR) data in the literature showed fraction I to be Lys-ligated, whereas the remaining fractions were reported to have an open $\left(\mathrm{OH}^{-} / \mathrm{H}_{2} \mathrm{O}\right.$ coordinated) distal site. ${ }^{37}$ All these observations support the view that CT-cyt $c$ shares structural features with previously characterized alkaline cyt $c$ conformers. ${ }^{19,34,36}$ Recent NMR and EPR work by Zhong et al. arrived at an analogous conclusion..$^{38}$

\section{Peroxidase activity of CT-cyt $c$ fractions}

Despite their similar CD and UV-Vis spectroscopic properties (Fig. S2 $\dagger$ ), the four CT-cyt $c$ fractions exhibited very different levels of peroxidase activity. Kinetic measurements were performed in the presence of $100 \mu \mathrm{M} \mathrm{H}_{2} \mathrm{O}_{2}$ (Fig. 2B and $\left.\mathrm{S} 3 \mathrm{~A}^{\dagger} \dagger\right)^{59}$
Fraction I showed a relatively low activity; that of fraction II was twofold higher, and for fraction III an additional $\sim$ twofold increase was observed. Fractions III and IV had similar activities. Under the low $\mathrm{H}_{2} \mathrm{O}_{2}$ concentration conditions of Fig. 2B, turnover was barely detectable for unmodified cyt $c$. This activity trend can be summarized as follows: unmodified cyt $c<$ fraction $\mathrm{I}<$ fraction $\mathrm{II}<$ fraction III $\approx$ fraction IV. The same trend was observed when the experiments were repeated at a higher $\mathrm{H}_{2} \mathrm{O}_{2}$ concentration of $500 \mu \mathrm{M}$, where even untreated cyt $c$ showed non-zero activity (Fig. $\mathrm{S} 3 \mathrm{~B} \dagger$ ).

A number of earlier studies implied that CT treatment of cyt $c$ generates a homogeneous product that is oxidized only at Met80 and Met65. ${ }^{\mathbf{1 7 , 4 8 , 5 0}}$ The data presented here demonstrate that this view is not correct. Instead, CT-cyt $c$ is a mixture of at least four species that can be separated by SCX (Fig. 2A) and that exhibit different peroxidase activities (Fig. 2B). While SCX fractionation of CT-cyt $c$ has been demonstrated previously, ${ }^{37,38,47,48}$ the chemical origin of this phenomenon and the associated activity differences remain unexplained. SCX separates proteins according to their cationic surface charge. CTinduced Met $\rightarrow$ MetO conversion does not directly alter the ionic nature of the protein (Fig. S4 $\dagger$ ). Conformational factors could, in principle, contribute to the SCX fractionation. ${ }^{48}$ However, the nearly indistinguishable CD and UV-Vis spectra point to similar overall structures for fractions I-IV (Fig. S2 $\dagger$ ), making conformational factors an unlikely culprit for the observed SCX behavior.

\section{SCX fractions represent specific proteoforms}

For uncovering the origin of the SCX properties and activity differences of fractions I-IV we applied a range of MS methods. As an initial step, intact protein mass spectra were acquired (Fig. 3). At first glance, the mass distributions of the four fractions appear to be almost identical, reminiscent of the optical data in Fig. S2. $\dagger$ Each spectrum in Fig. 3 is dominated by two signals that are spaced $+16 \mathrm{Da}$ apart, as expected for MetO
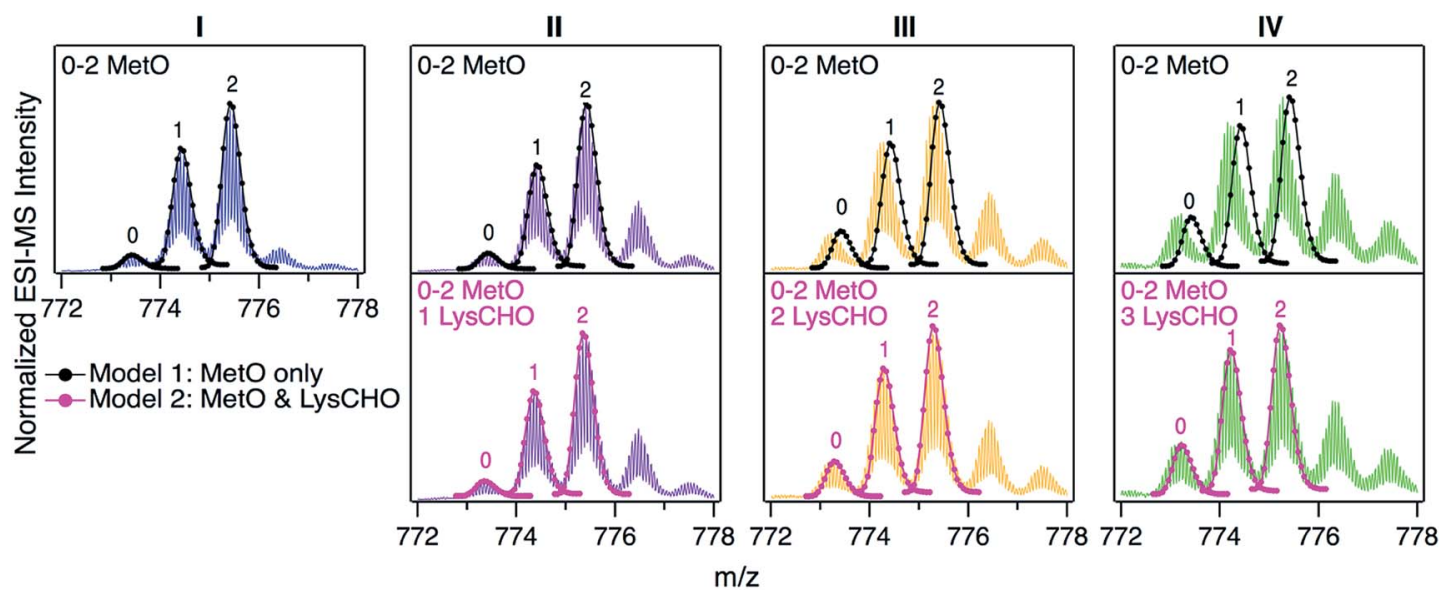

Fig. 3 Intact protein mass spectra of CT-cyt c fractions I-IV, focusing on the 16+ charge state, which yielded the highest signal intensity. Labels 0,1 , or 2 above peaks refer to the number of MetO sites (+16 Da). Top: Black lines/dots represent theoretical isotope distributions for MetO formation. Bottom: Magenta lines/dots represent theoretical isotope distributions for MetO formation plus one, two, or three LysCHO formation events (Lys carbonylation, $-1 \mathrm{Da}$ ). Top and bottom panels in each column show the same experimental data with different isotope models. 
formation at Met80 and/or Met65. In addition, each fraction showed a low intensity signal close to the mass of unmodified cyt $c$. In Fig. 3 these signals were annotated according to the number $(0,1,2)$ of MetO formation events. This interpretation reflects the existing literature, which assumes that CT specifically targets Met80 and Met65 while leaving other residues unmodified. ${ }^{17,37,38,47,48,50,51}$

To test the viability of this simple MetO-centric interpretation we calculated theoretical isotope distributions for unmodified cyt $c$, a +16 Da variant (one MetO), and a +32 Da variant (two MetO). These theoretical distributions were superimposed on the experimental spectra (Fig. 3, top row). Fraction I showed excellent agreement with the theoretical data. In contrast, fractions II, III, and IV exhibited gradually increasing mass defects, i.e., the measured peaks had a lower mass than expected. The magnitude of this discrepancy correlated with the SCX elution behavior: Fraction IV showed the largest discrepancy, while fraction I exhibited no discrepancy. The mismatch between experimental spectra and the theoretical "clean" +16 Da adducts implies that MetO formation is not the only type of covalent modification in fractions II-IV. Control experiments confirmed that mass discrepancies were already observable for unfractionated CT-cyt $c$, i.e., the observed effects were not an SCX-related artifact (Fig. S5 $\dagger$ ).

The missing piece of the puzzle that explains the properties of CT-cyt $c$ is Lys carbonylation (Fig. S4 $\dagger$ ). This reaction is known to be facilitated by metal centers (i.e., heme in cyt $c) .{ }^{43}$ It involves a radical intermediate ${ }^{63}$ that gets converted to $\varepsilon$-hydroxylysine. Elimination of ammonia then produces LysCHO. ${ }^{64}$ Each LysCHO formation event is accompanied by a -1 Da shift. ${ }^{41,64}$ Compilations of known oxidative protein modifications reveals LysCHO formation to be the only process associated with a -1 Da shift. ${ }^{42,43}$ The direct MS-based detection of LysCHO sites will be discussed below. Returning to Fig. 3, revised modeling of the mass spectra to account for a combination of MetO (+16 Da) and LysCHO formation $(-1 \mathrm{Da})$ resulted in excellent agreement between experimental and simulated spectra for all fractions (Fig. 3, bottom row). The isotope models suggest that fractions I, II, III, and IV carry $0,1,2$, and 3 LysCHO groups, respectively. These modifications are in addition to MetO formation at Met80 and Met65. ${ }^{17,37,38,47,48,50,51}$

The different numbers of LysCHO groups provide an explanation for the SCX behavior of the four fractions. The SCX strategy used here separates proteins on the basis of their overall cationic charge; each LysCHO site reduces this charge by one unit. ${ }^{41,42,64}$ In fraction I, the lack of Lys modifications causes the most positive surface charge, similar to that of unmodified cyt $c$ (pI 9.6). ${ }^{65}$ Hence, fraction I (and unmodified cyt $c$ ) interact most tightly with the SCX column, resulting in the largest elution volume (Fig. 2A). On the other extreme, fraction IV with its three LysCHO has lost three positive charges, causing those proteins to interact most weakly with the column. The behavior of fractions II and III falls in-between these two extremes.

In summary, LysCHO formation accounts for the previously unexplained observation ${ }^{37,38,47,48}$ that CT-cyt $c$ can be fractionated by SCX. LysCHO has been shown to play a role in the peroxidase activation of cyt $c$ by $\mathrm{H}_{2} \mathrm{O}_{2} \cdot{ }^{41}$ However, LysCHO formation in cyt $c$ has not been reported for oxidizing agents other than $\mathrm{H}_{2} \mathrm{O}_{2}$. The number of LysCHO sites is the only detectable difference in the covalent structures of fractions I-IV, implying that LysCHO formation must be responsible for their different activities. Combining the information of Fig. 1B and 3, the peroxidase activity can be ranked according to the number of LysCHO: fraction I (0 LysCHO) < fraction II (1 LysCHO) < fraction III ( 2 LysCHO) $\approx$ fraction IV (3 LysCHO).

\section{Top-down CID-IM-MS for proteoform-selective analysis}

Mapping of LysCHO sites poses significant analytical challenges. ${ }^{6}$ Typical bottom-up MS assays struggle with this task due to LysCHO-induced interference with tryptic cleavage and losses of ionization/fragmentation efficiency. ${ }^{42,67,68}$ Thus, it is not surprising that previous MS investigations failed to detect LysCHO in CT-cyt $c .^{17,18,37,38,45-48,50,51}$ To address these challenges we applied top-down MS/MS, i.e., gas phase fragmentation of undigested proteins. ${ }^{69}$ Initial attempts were unsuccessful due to the complexity of the fragment ion spectra. This problem was solved by incorporating ion mobility (IM) spectrometry to provide an additional separation dimension, thereby implementing a "top-down CID-IM-MS" workflow (Fig. S6†). Similar experiments have previously been applied to simple model systems ${ }^{70-72}$ but this work marks the first time that such an approach was used for tracking unknown biologically relevant modifications.

For validation, top-down CID-IM-MS was initially applied to peak 1 of fraction I (which is devoid of LysCHO) to examine the initial MetO formation event (Fig. 4). All C-terminal fragments starting at $\mathrm{y}_{25}$ showed a +16 Da shift relative to unmodified cyt $c$. The $\mathrm{y}_{25}$ to $\mathrm{y}_{24}$ transition allowed the oxidation site to be identified as Met80 with single-residue resolution. The presence of other oxidation sites in peak 1 (including Met65) could be ruled out. Measurements on peak $2(+32 \mathrm{Da})$ verified Met80 and Met65 as the only two oxidation sites in fraction I (Fig. S8 $\dagger$ ). Taken together, these data imply that CT-induced MetO formation is a sequential process, with Met80 as the initial oxidation target whereas Met65 is affected at a later stage.

\section{Mapping of LysCHO sites}

We next sought to identify the locations of LysCHO groups in fractions II, III, and IV by top-down CID-IM-MS. According to Fig. 3, these fractions possess 1, 2, and 3 carbonylation sites, respectively. LysCHO sites can be identified from -1 Da mass shifts of fragment ions. The experiments were facilitated by the fact that, fortuitously, peak 1 in all fractions corresponded exclusively to MetO formation at Met80 (Fig. S9†). Thus, LysCHO sites in the various fractions could be mapped via comparisons with fraction I fragments. By using this internal reference we could unequivocally rule out calibration errors as an explanation for the observed -1 Da shifts. Our assignments were further verified by simulated isotope distributions that were overlaid onto the experimental spectra.

Fig. 5 reveals that for all fractions, N-terminal fragments up to $\mathrm{b}_{46}$, as well as $\mathrm{C}$-terminal fragments up to $\mathrm{y}_{29}$ were indistinguishable from those of fraction I. This finding confines all 


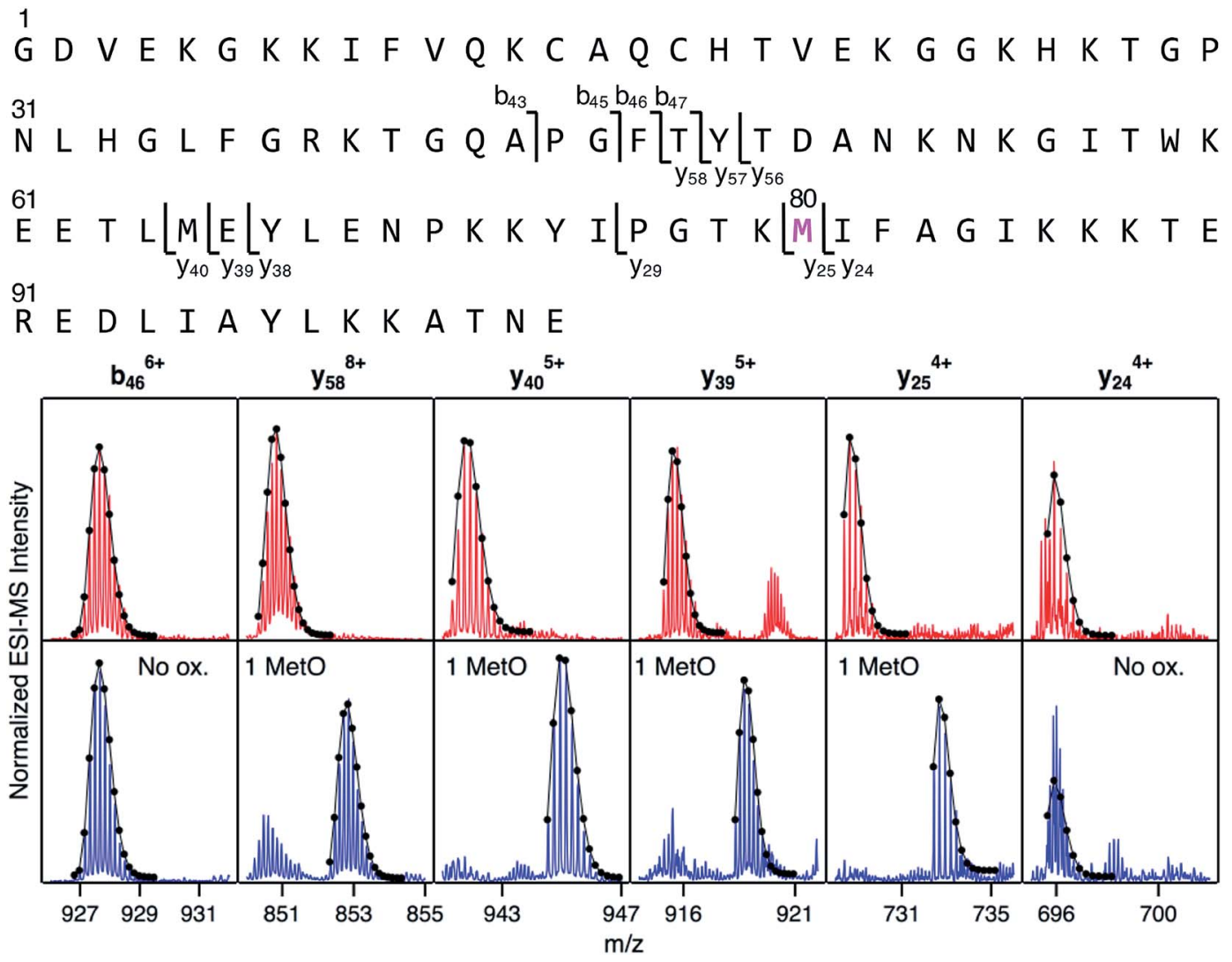

Fig. 4 Top-down CID-IM-MS data that pinpoint MetO formation in fraction I. The sequence is shown along the top, with key fragments indicated. Bottom: selected fragment ions from an unmodified control (red), and from peak 1 of fraction I (blue, see Fig. 3). Lines/dots represent theoretical isotope distributions calculated for unmodified fragments ("no ox."), or for MetO containing fragments. Met80 is the only covalent modification in peak 1 of fraction 1 .

LysCHO sites to residues $47-75$ which comprise five out of the total 19 Lys residues. In fraction II, $y$-ions from $y_{38}$ to $y_{58}$ showed a -1 Da shift. The $y_{38}$ to $y_{29}$ transition narrowed down the location of this modification to ${ }^{67} \mathrm{YLENPKK} \mathrm{YI}{ }^{75}$. Hence, the single LysCHO site in fraction II is located on Lys72 or Lys73 (Fig. 5, second row).

Fraction III also showed a $\mathrm{y}_{38}$ to $\mathrm{y}_{29}-1$ Da transition, implying one LysCHO modification on Lys72/73. An additional transition took place between $\mathrm{y}_{40}$ and $\mathrm{y}_{58}$, localizing the second LysCHO site to the region ${ }^{49}$ TDANKNKGITWKEETLM ${ }^{65}$. Thus, the second modification site in fraction III is located on Lys53, Lys55, or Lys60 (Fig. 5, third row).

Pinpointing the three LysCHO sites in Fraction IV was complicated by diminished fragmentation efficiencies that translated into low $\mathrm{S} / \mathrm{N}$ ratios. This phenomenon likely reflects the loss of protonation sites after LysCHO formation, keeping in mind that the proximity to $\mathrm{H}^{+}$sites favors $\mathrm{b} / \mathrm{y}$ ion formation. ${ }^{73-75}$ $\mathrm{y}_{58}$ exhibited a mass shift of -3 Da (Fig. 5, bottom row), confirming the presence of three LysCHO sites, as inferred from Fig. 3. From the fragment ion mass shifts in Fig. 5 (bottom row), the predominant modification pattern of fraction IV appears to be a single LysCHO in the Lys53/55/60 range, plus two LysCHO at Lys72/73. However, we cannot rule out that the final LysCHO formation event also affects Lys53/55/60 to some extent. The similar peroxidase activities of fractions III and IV suggest that this third LysCHO is of minor functional importance. Experiments analogous to those of Fig. 5 were conducted on peak 2 of each fraction, yielding LysCHO patterns similar to those of peak 1 (Fig. S10 and S11†).

Fig. 5 implies that CT-mediated LysCHO formation is a wellordered process. Starting from LysCHO-free protein (fraction I), carbonylation initially affects Lys72/73 (fraction II). The next carbonylation event takes place in the Lys53/55/60 region, generating fraction III. The final LysCHO site may affect Lys72/ 73 or Lys53/55/60. As noted above, MetO formation is also a sequential process, initially affecting Met80 and subsequently Met65.

\section{LC-MS/MS peptide mapping revisited}

The detection of LysCHO sites by top-down CID-IM-MS may seem at odds with previous studies on CT-cyt $c$ that only found MetO formation. ${ }^{17,37,38,47}$ In intact protein MS data the small ( -1 Da) mass shifts associated with LysCHO formation are easily overlooked, unless the spectra are carefully scrutinized via isotope modeling (Fig. 3). Similarly, LysCHO detection by traditional tryptic digestion LC-MS/MS (Fig. S1†) is difficult due to reduced fragmentation resulting from charge site loss, and suppression of tryptic cleavage. ${ }^{42,73-75}$ These issues are exacerbated for the situation encountered here, where up to three 

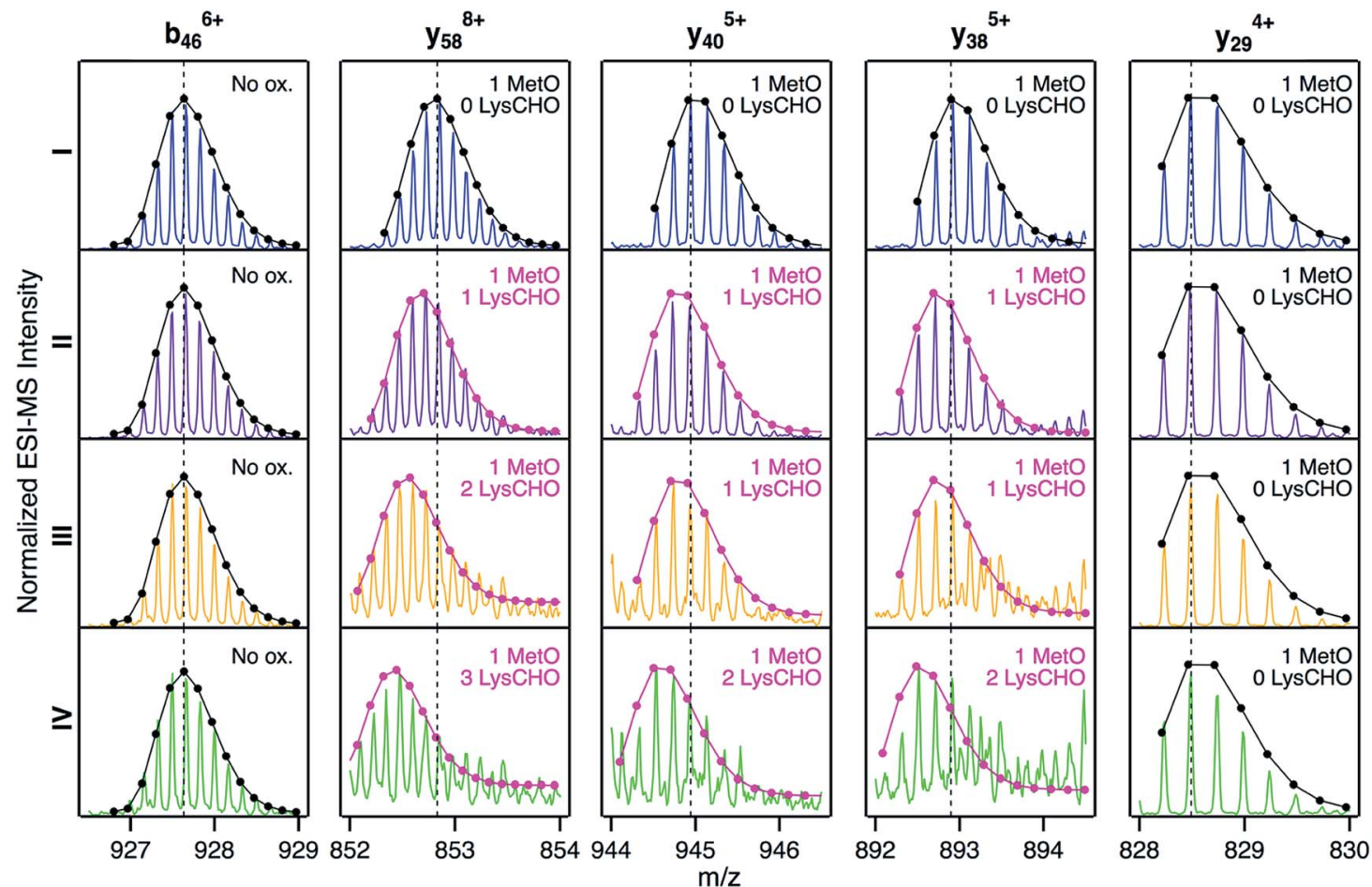

Fig. 5 Selected top-down CID-IM-MS ions generated by fragmentation of peak 1 from fractions I (blue), II (violet), III (orange), and IV (green). Each fragment ion is overlaid with its simulated isotopic envelope (lines/dots). Vertical dashed lines were included as visual aid to highlight LysCHO-related -1 Da mass shifts. Complete CID-IM-MS data are summarized in Fig. S7. $\uparrow$

LysCHO sites are confined to a fairly narrow sequence range (Lys53/55/60/72/73).

Armed with the information from top-down CID-IM-MS, we revisited the tryptic digestion LC-MS/MS workflow with careful optimization of experimental parameters. Gratifyingly, we were able to confirm carbonylation of Lys 72 in the missed-cleavage peptide T13-14 (residues 61-73, Fig. 6). Consistent with the data reported above, this LysCHO peptide was found in fractions II-IV, but not in fraction I. No other LysCHO sites were detected by LC-MS/MS. Thus, the top-down CID-IM-MS workflow is superior for this type of application, as it allowed the detection of all three carbonylation sites (Fig. 5).

\section{Mechanism of CT-induced peroxidase activation}

The data of this work provide a clear view of the sequential CTinduced events that convert cyt $c$ into an active peroxidase. This mechanism, summarized in Fig. 7, is governed by the undisputed fact that peroxidase activity requires the distal coordination site to be accessible. ${ }^{14,16,17}$

Unmodified (native) cyt $c$ has the lowest peroxidase activity of all the forms studied here because its distal ligation site is blocked by Met80. Very slow substrate turnover under these conditions may take place as the result of conformational fluctuations $^{52,53}$ that occasionally disrupt the Met80-Fe bond. ${ }^{40,41,76}$

CT-induced oxidation of Met80 generates fraction I, an "alkaline" conformer where Lys72 or Lys73 has moved into the ligation site previously occupied by Met80. This ligation switch is supported by RR data ${ }^{37}$ as well as NMR and EPR experiments. ${ }^{38}$ While the enhanced peroxidase activity of alkaline LysFe conformers is well documented, ${ }^{38}$ the origin of this effect is somewhat unclear because transient opening of the Lys-Fe contact takes place more slowly than for Met-Fe. ${ }^{34,77}$ Likely, the enhanced activity of fraction I is not caused by hexacoordinated Lys-Fe species per se, but by pentacoordinated structures that are part of the fraction I ensemble. ${ }^{14,38} \mathrm{RR}$ spectra are consistent with this interpretation, suggesting the presence of $\sim 15 \%$ pentacoordinated protein in the predominantly Lys-Fe coordinated fraction I ("peak A" in Fig. S13 of ref. 37). Other factors may contribute to the peroxidase activity of Lys-ligated cyt $c$ as well, ${ }^{41}$ one of these is the increased heme pocket volume which facilitates substrate access. ${ }^{19,77}$

As the next step in Fig. 7, carbonylation abrogates the iron ligating capability of Lys72/73, thereby generating fraction II. Fraction II still does not represent a genuine pentacoordinated conformer, evident from the fact that subsequent Lys53/55 carbonylation further enhances peroxidase activity (our MS data suggested the possible participation of Lys60, but we exclude this residue for reasons outlined below). Our data are thus consistent with a scenario where Lys53/55 participate in iron ligation in fraction II. However, these Lys53/55-Fe contacts must be quite labile, evident from the fact that fraction II has a higher activity than both fraction I and unmodified cyt $c$ (Fig. 2B).

Carbonylation of Lys53/55 subsequently produces fraction III which possesses a permanently open distal site and shows the highest peroxidase activity. RR data confirm that fraction III 


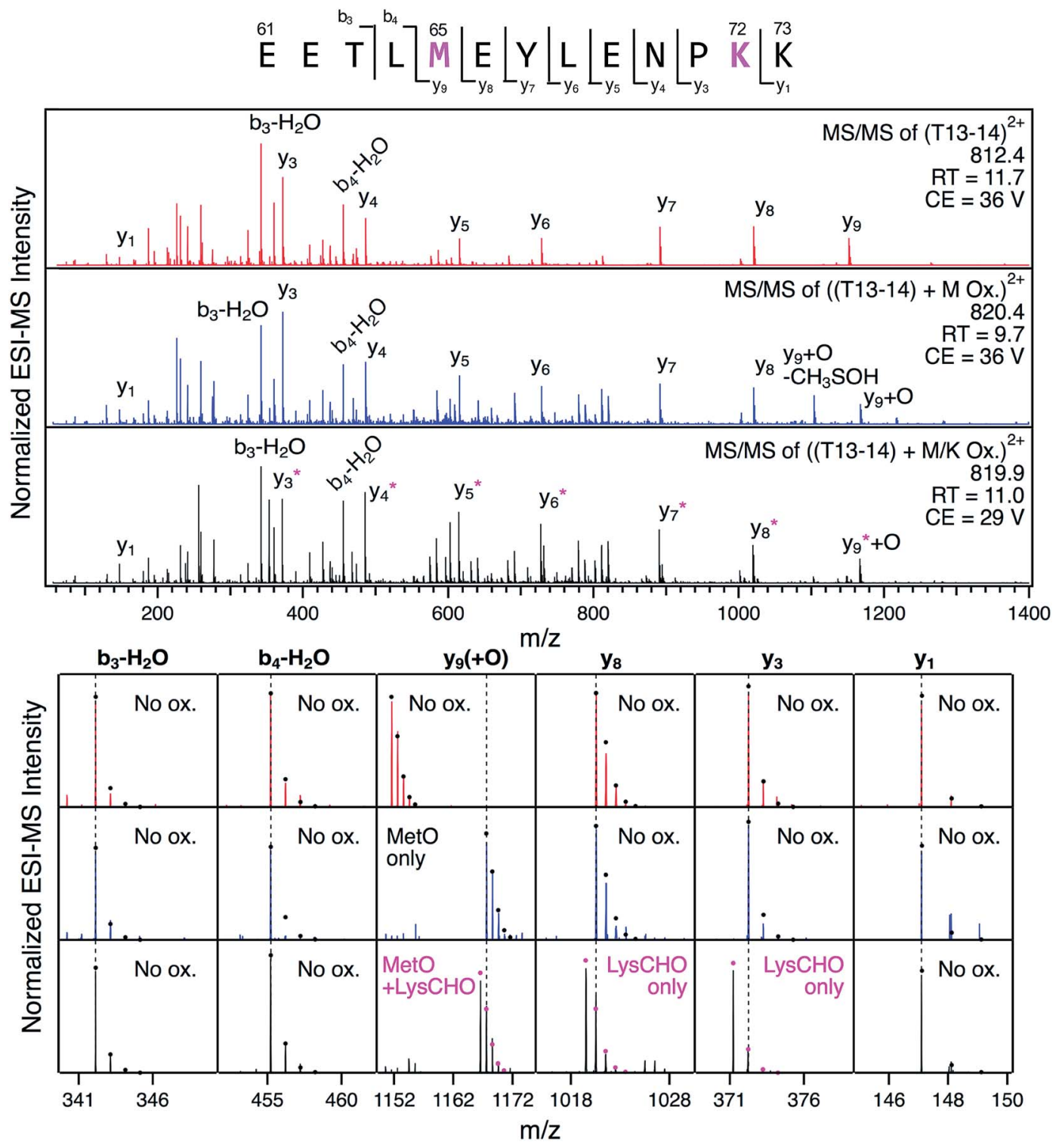

Fig. 6 LC-MS/MS analysis of tryptic peptide T13-14 ( ${ }^{61}$ EETLMEYLENPKK $\left.{ }^{73}\right)$ in unfractionated CT-cyt c. Three LC separated variants were interrogated: unmodified (red), with Met65 oxidation (blue), and with both Met65 oxidation and Lys72 carbonylation (black). Top: fragment ion spectra. Bottom: Magnified views of representative fragment ions, highlighting $+16 \mathrm{Da}$ (MetO) and $-1 \mathrm{Da}$ (LysCHO) mass shifts. lons denoted with an asterisk contain the LysCHO site. Simulated isotopic envelopes are shown as circles. Much lower collision energies (CE) were required for adequate fragmentation of the carbonylated peptide.

is pentacoordinated, as expected for a functional peroxidase. ${ }^{37} \mathrm{~A}$ final carbonylation event produces fraction IV which retains both an open distal site and peroxidase activity.

The essential point in the activation cascade of Fig. 7 is that CT-induced Met80 oxidation only results in a moderate level of peroxidase activity, because the distal site remains congested by Lys ligation. Subsequent CT-induced LysCHO formation frees up the distal site, thereby producing a genuine pentacoordinated heme. Previous work attributed the peroxidase activity of CT-cyt $c$ exclusively to MetO formation, ${ }^{\mathbf{1 7}, 18,37,38,44-47}$ failing to recognize the occurrence and mechanistic significance of Lys carbonylation. Our data reveal that MetO formation only represents an initial step en route toward peroxidase-activated
CT-cyt $c$. Lys carbonylation represents a co-activator that boosts the activity by a factor of $\sim 4$ (fractions III/IV) relative to the MetO-only form (fraction I, see Fig. 2).

The model of Fig. 7 provides a simple explanation for the correlation between the activity of the CT-cyt $c$ fractions and their SCX behavior: both factors are directly tied to the number of LysCHO sites. When accounting for the SCX fractionation behavior, one has to remember that while the protein interacts with the anionic stationary phase, it may adopt structures and ligation scenarios that differ from the bulk solution conformations of Fig. $7 .^{78}$

The ability of cyt $c$ to adopt various distal ligation scenarios is consistent with the foldon model that dissects the protein into 


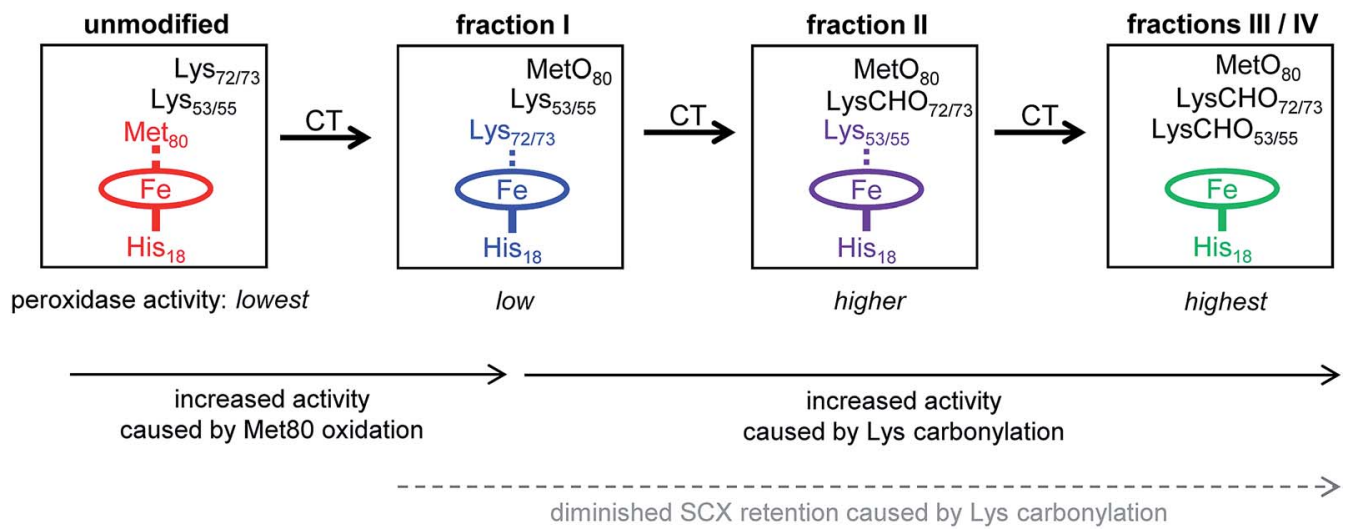

Fig. 7 Model of CT-induced peroxidase activation of cyt c. SCX fractions I-IV exhibit different activity due to distal ligation changes. In unmodified cyt $c$ the distal ligation site is blocked by Met80. In fraction I MetO formation has displaced Met80 from the heme iron, and distal ligation is provided by Lys72/73. In fraction II carbonylation of Lys72/73 causes a transition to distal ligation by Lys53/55. In fractions III and IV the heme has an open coordination site because carbonylation has abrogated the capability of Lys72/73 and Lys53/55 to act as distal ligands. Peroxidase activity increases from left to right, concomitant with increased accessibility of the distal ligation site. Dashed lines indicate Fe coordination bonds that can transiently open as a result of protein conformational fluctuations.

regions of different stability (Fig. S12 $\dagger$ ). ${ }^{52}$ The gray and red foldons (40-57 and 71-85 $\Omega$ loops, respectively) show the highest flexibility. ${ }^{52}$ Not surprisingly, the alternative Fe ligands identified above are located in these two flexible regions, i.e., Lys72/73 (red) and Lys53/55 (gray). Opening of gray $^{79}$ and red $^{53}$ has previously been linked to alkaline conformers. The flexibility of gray and red also governs the CT-induced transitions outlined in Fig. 7. In native cyt $c$ the red foldon is anchored by the Met80Fe linkage. ${ }^{52,53,79}$ Our data suggest that MetO formation sterically perturbs this (already labile) ${ }^{52}$ region, thereby triggering rearrangements that allow Lys72/73 to move into the distal site. ${ }^{53}$ The foldon hierarchy dictates that red can only open up when gray is already unfolded. ${ }^{52,53,79}$ Hence, after carbonylation of Lys72/73, the plasticity of the unfolded gray foldon will allow Lys53/55 to move into the vacated distal site. Our MS data suggest that Lys60 could also participate in Fe ligation, but its placement in the relatively rigid yellow foldon renders such a scenario less likely (Fig. S12†).

Our data do not provide evidence for carbonylation of Lys79. In principle, this residue represents another possible ligand that might block access to the iron after Met80 oxidation. ${ }^{38}$ However, evidence for such Lys79-Fe contacts comes primarily from work on K72A yeast iso-1 cyt $c$ which has coordination propensities that are different from those of the horse wild type studied here. ${ }^{38}$ Our findings suggest that Lys79-Fe contacts do not play a major role in Met80-oxidized horse cyt $c$, such that peroxidase activation is attainable without Lys79 carbonylation.

\section{Conclusions}

Our discovery of multiple, previously unreported carbonylation events in CT-cyt $c$ calls into question the prevailing Met80centric view of peroxidase activation, according to which oxidation of Met80 alone is sufficient for turning cyt $c$ into a pentacoordinated peroxidase. ${ }^{17,18,37,38,44,45,47,48}$ The conclusions from a number of those reports have relied on the alleged fidelity of CT for specific Met oxidation. Several studies pooled the highest intensity region in the SCX chromatogram, assuming that samples obtained in this way represent the expected "clean" MetO species. ${ }^{37,38,47,48}$ Our data strongly suggest that such samples correspond to fractions II/III, with oxidation at Met80, Met65, and with LysCHO sites in the Lys72/73 and Lys53/55 range. Carbonylation of these samples enhances their peroxidase activity almost fourfold relative to fraction I, which represents the true MetO-only species (Fig. 2 and 7). It appears that past studies inadvertently studied carbonylated CT-cyt $c$ proteoforms, misattributing some of the carbonylation-induced effects to MetO formation.

Previous studies on CT-cyt $c$ yielded conflicting results, suggesting that the distal site is open ${ }^{37}$ or occupied by Lys. ${ }^{38}$ The present work helps resolve these discrepancies. CT-cyt $c$ comprises multiple species with different coordination patterns (Fig. 7). Thus, any results will depend on the precise experimental conditions used for isolating protein from the CT-cyt $c$ reaction mixture.

Only four out of the 19 Lys residues in cyt $c$ were identified as possible carbonylation sites. In native cyt $c$ these four residues are relatively close to the distal face of the heme, which represents the site of peroxidase catalysis (Fig. 1). Considering that all 19 Lys residues are solvent-exposed (Fig. S13†), the spatial selectivity of LysCHO formation rules out diffusion-mediated oxidation events, where modifications are governed by solvent accessibility. ${ }^{55}$ It instead appears that LysCHO formation is heme-dependent. ${ }^{43,58}$ This line of thought bolsters our conclusions, as carbonylation of Lys53/55/72/73 confirms the capability of these residues to come in direct contact with the heme by serving as alternative Fe ligands (Fig. 7).

There are certain parallels, but also differences between the CT-induced peroxidase activation of cyt $c$ studied here, and the $\mathrm{H}_{2} \mathrm{O}_{2}$-induced activation that we explored earlier. ${ }^{41}$ The initial transformation triggered by $\mathrm{H}_{2} \mathrm{O}_{2}$ is oxidation of Tyr67, while this residue remains unmodified during $\mathrm{CT}$ exposure. Peroxidase 
activation by both CT and $\mathrm{H}_{2} \mathrm{O}_{2}$ involves sequential modifications and transiently populated alkaline conformers, ultimately producing a vacant distal site. One key difference is that CTinduced activation relies on MetO formation for disruption of the Met80-Fe bond (Fig. 7). In contrast, $\mathrm{H}_{2} \mathrm{O}_{2}$ causes this bond to break as a result of Tyr67 oxidation, without Met80 oxidation. The reasons underlying the different initial reactivities of the two oxidants remain to be fully explored. Likely, $\mathrm{H}_{2} \mathrm{O}_{2}$ oxidation of Tyr67 proceeds with catalysis by the iron center, ${ }^{43}$ whereas CT can oxidize Met without heme involvement. ${ }^{51}$ A common feature of CT and $\mathrm{H}_{2} \mathrm{O}_{2}$-induced activation is LysCHO formation which prevents the distal site from being blocked by Lys side chains. It is likely that LysCHO formation plays a similar role for the conversion of cyt $c$ into an apoptotic peroxidase under other conditions, e.g., after cardiolipin binding ${ }^{3,27-29}$ in vitro and in vivo. However, this hypothesis remains to be verified experimentally.

\section{Conflicts of interest}

There are no conflicts to declare.

\section{Acknowledgements}

Funding was provided by the Natural Sciences and Engineering Research Council of Canada (RGPIN-2018-04243).

\section{References}

1 D. Alvarez-Paggi, L. Hannibal, M. A. Castro, S. Oviedo-Rouco, V. Demicheli, V. Tórtora, F. Tomasina, R. Radi and D. H. Murgida, Chem. Rev., 2017, 117, 13382-13460.

2 R. Radi, L. M. Thomson, H. Rubbo and E. Prodanov, Arch. Biochem. Biophys., 1991, 288, 112-117.

3 V. E. Kagan, H. A. Bayır, N. A. Belikova, O. Kapralov, Y. Y. Tyurina, V. A. Tyurin, J. Jiang, D. A. Stoyanovsky, P. Wipf, P. M. Kochanek, J. S. Greenberger, B. Pitt, A. A. Shvedova and G. G. Borisenko, Free Radical Biol. Med., 2009, 46, 1439-1453.

4 L. C. Godoy, C. Muñoz-Pinedo, L. Castro, S. Cardaci, C. M. Schonhoff, M. King, V. Tórtora, M. Marín, Q. Miao, J. F. Jiang, A. A. Kapralov, R. Jemmerson, G. G. Silkstone, J. N. Patel, J. E. Evans, M. 1. T. Wilson, D. R. Green, V. E. Kagan, R. Radi and J. B. Mannick, Proc. Natl. Acad. Sci. U. S. A., 2009, 106, 2653-2658.

5 L. Hannibal, F. Tomasina, D. A. Capdevila, V. Demicheli, V. Tórtora, D. Alvarez-Paggi, R. Jemmerson, D. H. Murgida and R. Radi, Biochemistry, 2016, 55, 407-428.

6 M. D. Liptak, R. D. Fagerlund, E. C. Ledgerwood, S. M. Wilbanks and K. L. Bren, J. Am. Chem. Soc., 2011, 133, 1153-1155.

7 L. J. McClelland, T.-C. Mou, M. E. Jeakins-Cooley, S. R. Sprang and B. E. Bowler, Proc. Natl. Acad. Sci. U. S. A., 2014, 111, 6648-6653.

8 C. M. Jenkins, K. Yang, G. Liu, S. H. Moon, B. G. Dilthey and

R. W. Gross, J. Biol. Chem., 2018, 293, 8693-8709.

9 N. C. Veitch, Phytochemicals, 2004, 65, 249-259.
10 J. P. Kitt, D. A. Bryce, S. D. Minteer and J. M. Harris, J. Am. Chem. Soc., 2017, 139, 3851-3860.

11 G. Oemer, K. Lackner, K. Muigg, G. Krumschnabel, K. Watschinger, S. Sailer, H. Lindner, E. Gnaiger, S. B. Wortmann, E. R. Werner, J. Zschocke and M. A. Keller, Proc. Natl. Acad. Sci. U. S. A., 2018, 115, 41584163.

12 N. A. Belikova, Y. A. Vladimirov, A. N. Osipov, A. A. Kapralov, V. A. Tyurin, M. V. Potapovich, L. V. Basova, J. Peterson, I. V. Kurnikov and V. E. Kagan, Biochemistry, 2006, 45, 4998-5009.

13 R. S. Y. Wong, J. Exp. Clin. Cancer Res., 2011, 30.

14 R. E. M. Diederix, M. Ubbink and G. W. Canters, Biochemistry, 2002, 41, 13067-13077.

15 G. W. Bushnell, G. V. Louie and G. D. Brayer, J. Mol. Biol., 1990, 214, 585-595.

16 K. L. Bren and E. L. Raven, Science, 2017, 356, 1236.

17 Y.-R. Chen, L. J. Deterding, B. E. Sturgeon, K. B. Tomer and R. P. Mason, J. Biol. Chem., 2002, 277, 29781-29791.

18 Z. Wang, Y. Ando, A. D. Nugraheni, C. Ren, S. Nagao and S. Hirota, Mol. BioSyst., 2014, 10, 3130-3137.

19 J. F. Amacher, F. F. Zhong, G. P. Lisi, M. Q. Zhu, S. L. Alden, K. R. Hoke, D. R. Madden and E. V. Pletneva, J. Am. Chem. Soc., 2015, 137, 8435-8449.

20 L. A. Abriata, A. Cassina, V. Tortora, M. Marin, J. M. Souza, L. Castro, A. J. Vila and R. Radi, J. Biol. Chem., 2009, 284, 17-26.

21 T. M. Josephs, I. M. Morison, C. L. Day, S. M. Wilbanks and E. C. Ledgerwood, Biochem. J., 2014, 458, 259-265.

22 A. I. Karsisiotis, O. M. Deacon, M. T. Wilson, C. Macdonald, T. M. A. Blumenschein, G. R. Moore and J. A. R. Worrall, Sci. Rep., 2016, 6, 30447.

23 B. Moreno-Beltran, A. Guerra-Castellano, A. Diaz-Quintana, R. Del Conte, S. M. Garcia-Maurino, S. Diaz-Moreno, K. Gonzalez-Arzola, C. Santos-Ocana, A. Velazquez-Campoy, M. A. De la Rosa, P. Turano and I. Diaz-Moreno, Proc. Natl. Acad. Sci. U. S. A., 2017, 114, E3041-E3050.

24 L. Tognaccini, C. Ciaccio, V. D'Oria, M. Cervelli, B. D. Howes, M. Coletta, P. Mariottini, G. Smulevich and L. Fiorucci, J. Inorg. Biochem., 2016, 155, 56-66.

25 C. Ciaccio, L. Tognaccini, T. Battista, M. Cervelli, B. D. Howes, R. Santucci, M. Coletta, P. Mariottini, G. Smulevich and L. Fiorucci, J. Inorg. Biochem., 2017, 169, 86-96.

26 Z.-H. Wang, Y.-W. Lin, F. I. Rosell, F.-Y. Ni, H.-J. Lu, P.-Y. Yang, X.-S. Tan, X.-Y. Li, Z.-X. Huang and A. G. Mauk, ChemBioChem, 2007, 8, 607-609.

27 M. Abe, R. Niibayashi, S. Koubori, I. Moriyama and H. Miyoshi, Biochemistry, 2011, 50, 8383-8391.

28 L. Serpas, B. Milorey, L. A. Pandiscia, A. W. Addison and R. Schweitzer-Stenner, J. Phys. Chem. B, 2016, 120, 1221912231.

29 J. M. Bradley, G. Silkstone, M. T. Wilson, M. R. Cheesman and J. N. Butt, J. Am. Chem. Soc., 2011, 133, 19676-19679.

30 M. Assfalg, I. Bertini, A. Dolfi, P. Turano, A. G. Mauk, F. I. Rosell and H. B. Gray, J. Am. Chem. Soc., 2003, 125, 2913-2922. 
31 S. M. Nold, H. Lei, T.-C. Mou and B. E. Bowler, Biochemistry, 2017, 56, 3358-3368.

32 J. M. García-Heredia, I. Díaz-Moreno, P. M. Nieto, M. Orzáez, S. Kocanis, M. Teixeira, E. Pérez-Payá, A. Díaz-Quintana and M. A. De la Rosa, Biochim. Biophys. Acta, 2010, 1797, 981-993.

33 S. Döpner, P. Hildebrandt, F. I. Rosell and A. G. Mauk, J. Am. Chem. Soc., 1998, 120, 11246-11255.

34 F. I. Rosell, J. C. Ferrer and A. G. Mauk, J. Am. Chem. Soc., 1998, 120, 11234-11245.

35 J. Gu, D.-W. Shin and E. V. Pletneva, Biochemistry, 2017, 56, 2950-2966.

36 J. M. Garcia-Heredia, A. Diaz-Quintana, M. Salzano, M. Orzaez, E. Perez-Paya, M. Teixeira, M. A. De la Rosa and I. Diaz-Moreno, J. Biol. Inorg Chem., 2011, 16, 1155-1168.

37 D. A. Capdevila, W. A. Marmisolle, F. Tomasina, V. Demicheli, M. Portela, R. Radi and D. H. Murgida, Chem. Sci., 2015, 6, 705-713.

38 F. Zhong and E. V. Pletneva, Inorg. Chem., 2018, 57, 57545766.

39 D. A. Capdevila, D. Alvarez-Paggi, M. A. Castro, V. Tortora, V. Demicheli, D. A. Estrin, R. Radi and D. H. Murgida, Chem. Commun., 2014, 50, 2592-2594.

40 O. M. Deacon, D. A. Svistunenko, G. R. Moore, M. T. Wilson and J. A. R. Worrall, Biochemistry, 2018, 57, 4276-4288.

41 V. Yin, G. S. Shaw and L. Konermann, J. Am. Chem. Soc., 2017, 139, 15701-15709.

42 A. Bachi, I. Dalle-Donne and A. Scaloni, Chem. Rev., 2013, 113, 596-698.

43 I. M. Møller, A. Rogowska-Wrzesinska and R. S. P. Rao, J. Proteomics, 2011, 74, 2228-2242.

44 G. H. Lushington, A. B. Cowley, S. Silchenko, G. S. LukatRodgers, K. R. Rodgers and D. R. Benson, Inorg. Chem., 2003, 42, 7550-7559.

45 A. V. Birk, W. M. Chao, S. Y. Liu, Y. Soong and H. H. Szeto, Biochim. Biophys. Acta, 2015, 1847, 1075-1084.

46 A. D. Nugraheni, C. G. Ren, Y. Matsumoto, S. Nagao, M. Yamanaka and S. Hirota, J. Inorg. Biochem., 2018, 182, 200-207.

47 R. D. Parakra, G. N. L. Kleffmann, T. Jameson and E. C. Ledgerwood, Dalton Trans., 2018, 47, 9128-9135.

48 J. Pande, K. Kinnally, K. K. Thallum, B. C. Verma, Y. P. Myer, L. Rechsteiner and H. R. Bosshard, J. Protein Chem., 1987, 6, 295-319.

49 S. P. Mushran, M. C. Agrawal and B. Prasad, J. Chem. Soc. B, 1971, 1712-1714.

50 B. Michel, A. E. I. Proudfoot, C. J. A. Wallace and H. R. Bosshard, Biochemistry, 1989, 28, 456-462.

51 Y. Shechter, Y. Burstein and A. Patchornik, Biochemistry, 1975, 14, 4497-4503.

52 W. B. Hu, Z. Y. Kan, L. Mayne and S. W. Englander, Proc. Natl. Acad. Sci. U. S. A., 2016, 113, 3809-3814.

53 H. Maity, J. N. Rumbley and S. W. Englander, Proteins, 2006, 63, 349-355.
54 L. M. Jones, J. B. Sperry, J. A. Carroll and M. L. Gross, Anal. Chem., 2011, 83, 7657-7661.

55 G. Xu and M. R. Chance, Chem. Rev., 2007, 107, 3514-3543.

56 M. Kathiresan and A. M. English, Chem. Sci., 2017, 8, 11521162.

57 J. S. Sharp, J. M. Becker and R. L. Hettich, Anal. Chem., 2004, 76, 672-683.

58 J. D. Bridgewater, J. Lim and R. W. Vachet, Anal. Chem., 2006, 78, 2432-2438.

59 D. A. Baldwin, H. M. Marques and J. M. Pratt, J. Inorg. Biochem., 1987, 30, 203-217.

60 M. Giorgio, M. Trinei, E. Migliaccio and P. G. Pelicci, Nat. Rev. Mol. Cell Biol., 2007, 8, 722A-728A.

61 S. W. Kelly, T. J. Jess and N. C. Price, Biochim. Biophys. Acta, 2005, 1751, 119-139.

62 L. S. Kaminsky, V. J. Miller and A. J. Davison, Biochemistry, 1973, 12, 2215-2221.

63 Y. R. Chen, C. L. Chen, X. P. Liu, H. T. Li, J. L. Zweier and R. P. Mason, Free Radical Biol. Med., 2004, 37, 1591-1603.

64 J. R. Requena, C.-C. Chao, R. L. Levine and E. R. Stadtman, Proc. Natl. Acad. Sci. U. S. A., 2001, 98, 69-74.

65 M. Graf, R. G. Garcia and H. Watzig, Electrophoresis, 2005, 26, 2409-2417.

66 W. G. Chung, C. L. Miranda and C. S. Maier, Electrophoresis, 2008, 29, 1317-1324.

67 A. Temple, T.-Y. Yen and S. Gronert, J. Am. Soc. Mass Spectrom., 2006, 17, 1172-1180.

68 K. Artemenko, J. Mi and J. Bergquist, Free Radical Res., 2015, 49, 477-493.

69 N. Siuti and N. L. Kelleher, Nat. Methods, 2007, 4, 817-821.

70 N. F. Zinnel, P.-J. Pai and D. H. Russell, Anal. Chem., 2012, 84, 3390-3397.

71 D. A. Polasky, F. Lermyte, M. Nshanian, F. Sobott, P. C. Andrews, J. A. Loo and B. T. Ruotolo, Anal. Chem., 2018, 90, 2756-2764.

72 F. Halgand, J. Habchi, L. Cravello, M. Martinho, B. Guigliarelli and S. Longhi, Anal. Chem., 2011, 83, 73067315.

73 A. R. Dongré, J. L. Jones, Á. Somogyi and V. H. Wysocki, J. Am. Chem. Soc., 1996, 118, 8365-8374.

74 B. Paizs and S. Suhai, Mass Spectrom. Rev., 2005, 24, 508-548. 75 S. M. Greer, D. D. Holden, R. Fellers, N. L. Kelleher and J. S. Brodbelt, J. Am. Soc. Mass Spectrom., 2017, 28, 15871599.

76 N. Tomášková, R. Varhač, V. Lysáková, A. Musatov and E. Sedlák, Biochim. Biophys. Acta, 2018, 1866, 1073-1083.

77 Y. Deng, F. Zhong, S. L. Alden, K. R. Hoke and E. V. Pletneva, Biochemistry, 2018, 57, 5827-5840.

78 J. Guo and G. Carta, J. Chromatogr. A, 2015, 1388, 184-194.

79 M. M. G. Krishna, Y. Lin, J. N. Rumbley and S. W. Englander, J. Mol. Biol., 2003, 331, 29-36. 